\title{
DIGITAL SIGNAL PROCESSING FOR PREDICTING STOCK PRICES
}

\section{Bello Abdulazeez Omeiza ${ }^{1}$, and Kabari Ledisi G. ${ }^{2}$}

Computer Science Department, Faculty of Natural Science and Applied Sciences, Ignatius Ajuru University of Education.

${ }^{1}$ Email: bello2527@gmail.com,

${ }^{2}$ Email: ledisigiokkabari@yahoo.com

Cite this article:

Bello A.O., Kabari L.G. (2021), Digital Signal Processing for Predicting Stock Prices. British Journal of Computer, Networking and Information Technology 4(2), 12-21. DOI 10.52589/BJCNIT-

XNP3UBPL.

\section{Manuscript History}

Received: 15 July 2021

Accepted: 24 Aug 2021

Published: 5 Sept 2021

Copyright $\left({ }_{0} 2020\right.$ The Author(s). This is an Open Access article distributed under the terms of Creative Commons AttributionNonCommercial-NoDerivatives 4.0 International (CC BY-NC-ND

4.0 ), which permits anyone to share, use, reproduce and redistribute in any medium, provided the original author and source are credited.
ABSTRACT: With the exponential growth of big data and data warehousing, the amount of data collected from various stock markets around the world has increased significantly. It is now impossible to process and analyze data using mathematical techniques and basic statistical calculations to forecast trends such as closing and opening prices, as well as daily stock market lows and highs. The development of smart and automated stock market forecasting systems has made significant progress in recent years. Digital signal processing is required for analysis and preprocessing because of the accuracy and speed with which these large amounts of data must be processed and analyzed. In this paper, we evaluate some of these predictive algorithms based on three parameters such as speed, accuracy and complexity, we analyze the data using the dataset from kaggle.com and we implement these algorithms using pythons. The results of our analysis in this paper shows a significant correlation between the yearly prices until the year 2018 where there is a significant increase in stock price.

KEYWORDS: Digital Signal Processing, Forecasting, Prediction, Predictive algorithm, Stock Market 


\section{INTRODUCTION}

Stock index forecasting has always been a fascinating and pressing topic. Predicting stock market behaviour using various techniques and methods is a useful tool for investors to act with greater certainty, taking into account the risks and volatility of an investment, and knowing when to buy at the lowest price and sell at the highest price. Increases and decreases in stock market prices are influenced by a variety of factors such as demand, exchange rates, gold and oil prices, political and economic events, and so on. On the other hand, we can look at stock market price variation as a time series and ignore the aforementioned factors.

The upward and downward movement of stock market prices is influenced by several factors, including the amount of demand, the exchange rate, the price of oil, as well as political and economic trends. Price dispersion in the stock market can be viewed as a time series, and price predictions can be made in the future simply by determining the sequence rules of the price train. When new information is released in the financial market, predictive tools can be used on historical stock data to forecast the future.

Nonetheless, because most stock market data can be viewed as discrete signs (values examined at regular intervals such as hourly, daily, monthly, or yearly), it was relatively easy to adapt existing channel strategies for financial exchange analysis. In any case, the calculations must include the following two primary characteristics:

- Accuracy was critical because most stock market predictions must be made in real-time and must be accurate and precise; otherwise, major financial losses could result. To achieve this, the filters must be dynamic, able to adapt to a variety of factors and changes in real-time and make predictions accordingly. This necessitated the use of feedback in prediction algorithms, which led to the development of Adaptive Filters.

- $\quad$ Speed and ability to process large amounts of data: Stock market trends are influenced by a variety of factors such as stock price history, previous day closing prices, opening prices, daily highs and lows, as well as external factors such as market trends of different countries, and so on. All of this results in a large amount of data that must be processed in a short amount of time in order to make accurate predictions.

The paper concentrate on the application of adaptive filters to the study of stock market trends. Adaptive filters are recursive, and they use feedback to correct errors. This qualifies them to make accurate predictions because they can account for market volatility. For the stock market, data is typically available in the form of charts (histograms, pie charts, etc.) or as a time series.

Stock file prediction has always been a tempting and enticing field. Anticipating financial exchange behaviour through strategies and various techniques is a valuable tool to assist speculators in acting with more prominent certainty, confronting the challenges and unpredictability of a venture into thought, and understanding when to purchase the least expensive cost and when to offer to the most exorbitant cost. Increases and decreases in financial exchange costs are influenced by a variety of factors, such as interest rate, conversion scale, gold price, oil price, political and monetary events; however, from another perspective, we can consider the securities exchange cost variation as a time arrangement and without reference to the aforementioned variables, and only by determining the succession rules of cost. The goal here is to achieve a reasonable level of accuracy by employing two different prediction models and then comparing the results using the mean squared error. The variable 
being predicted is today's closing price and opening price using either the previous day's opening, low, high and adjusted close price or using a time series of the closing prices. The first step is to collect and analyze large amounts of historical data. Data scraping is a concept that has been used. The Gradient descent/Steepest descent algorithm is the first. This linear regression algorithm seeks to generate a hypothesis function that is as close to the correct output signal as possible. Linear regression is, at its core, curve fitting. A curve is plotted using the set of input training data, the equation of which is the hypothesis function. The cost of work is the mean squared difference between the expected and actual yield. The limited cost function aids in weight updating and hypothesis function improvement. The following algorithm is a simplified version of Prony's Normal Equation algorithm, which can register the arrangement in a single step, whereas inclination drop would require multiple steps. All shaft frameworks have a little more computational flexibility than the broader post zero models. The shafts are used to make the Normal Equation larger. The posts are discovered in order to reduce the squared error. The model is especially useful for forecasting transient prices. Financial analysts who invest in the stock market are frequently unaware of market trends. They're having trouble trading because they don't fully understand which stocks to buy or sell to make a profit. In today's world, all stock market information is readily available. Analyzing all of this data individually or manually is extremely difficult. This is where data mining techniques come in handy. Understanding that numerical time series analysis yields close results, intelligent investors use machine learning techniques to forecast stock market behaviour. This will enable financial analysts to predict the behaviour of the stock in question and act accordingly.

Because the price is everything in the stock market and is influenced by many factors such as season, change of government, policymakers' decisions, and so on, there is a need for a system capable of predicting future prices with some degree of accuracy. This paper tends to address the problem of using traditional statistics and mathematical techniques in analysing data and predicting trends like the stock market. This paper aims to develop a digital signal processing for predicting stock prices using python. We use data from kaggle.com to create input to our system and we apply python programming language for implementation.

\section{LITERATURE REVIEW}

\section{Digital Signal Processing}

Digital signal processing is the mathematical manipulation of an information signal, such as audio, temperature, voice, or video, to modify or improve it in some way. DSP filters, measures, compresses and produces analogue signals by manipulating various types of signals. Digital signals take information and convert it into binary format, where each bit of data is represented by two distinct amplitudes. Analogue signals take information and convert it into varying amplitude electric pulses, whereas digital signals take information and convert it into binary format, where each bit of data is represented by two distinct amplitudes. Analogue signals can be represented as sine waves, whereas digital signals can be represented as square waves. DSP is used in almost every field, including oil processing, sound reproduction, radar and sonar, medical image processing, and telecommunications - basically, any application that compresses and reproduces signals (Donald, 2015) 


\section{Stock Market Prediction}

The act of attempting to predict the future value of company stock or other financial instrument traded on an exchange is known as the stock market prediction. A successful forecast of a stock's future price could result in a large profit. Stock prices, according to the efficient-market hypothesis, reflect all currently available information, and any price changes that are not based on newly revealed information are thus inherently unpredictable. Others disagree, and those who hold this viewpoint claim to have a variety of methods and technologies that allow them to obtain future price information (Wikipedia, 2009). According to the efficient market hypothesis, stock prices are a function of information and rational expectations, and newly revealed information about a company's prospects is reflected almost immediately in the current stock price. This would imply that all publicly available information about a company, including its price history, is already reflected in the stock's current price. As a result, stock price changes reflect the release of new information, changes in the market as a whole, or random movements around a value that reflects the existing information set. In his influential 1973 book A Random Walk Down Wall Street, Burton Malkiel claimed that stock prices could not be predicted accurately by looking at price history. As a result, according to Malkiel, stock prices are best described by a statistical process known as a "random walk," which implies that daily deviations from the central value are random and unpredictable. As a result, Malkiel came to the conclusion that paying financial services professionals to forecast the market harmed, rather than helped, net portfolio return. Several empirical tests back up the theory's general applicability, as most professional stock predictors' portfolios do not outperform the market average return.

Sharma and Kaushik's (2018) research centred on attempting to answer the question of whether or not real stock prices can be forecasted - the forecastability of real stock prices. The research employed what he referred to as the martingale hypothesis, which primarily considered the predictability of price changes or returns. Finally, the study took into account efficient market theory. As a result, stock market prices are a reliable variable that can be influenced by a simple social media tweet.

Granger (1992) looked at a variety of methods and algorithms for forecasting stock market prices. While each algorithm and approach has its own set of advantages, it was discovered that the Long Short Term Memory (LSTM) and Neural Network methods outperformed the others.

Singh (2018) developed a predicting system by combining machine learning and deep learning techniques with Python code. Different algorithms, such as k-Nearest Neighbours, Linear Regression, and moving average, were used in the research.

\section{RESEARCH METHODOLOGY}

Recent stock market data were collected from www.kaggle.com. The data contain the date of the market activity, opening and closing stock, highest and lowest stock and volume. We analyzed the data using digital signal processing with the help of the python programming language for implementation. By plotting the stocks in form of signals, we were able to predict stock price. Table 1 shows the data set. 
British Journal of Computer, Networking and Information Technology

ISSN: 2689-5315

Volume 4, Issue 2, 2021 (pp. 12-21)

www.abjournals.org

Table 1: Data Set from www.kaggle.com

\begin{tabular}{|c|c|c|c|c|c|c|}
\hline Date & Open & High & Low & Close & Close & Volume \\
\hline $1 / 2 / 2018$ & 1048.34 & 1066.94 & 1045.23 & 1065 & 1065 & 1237600 \\
\hline $1 / 3 / 2018$ & 1064.31 & 1086.29 & 1063.21 & 1082.48 & 1082.48 & 1430200 \\
\hline $1 / 4 / 2018$ & 1088 & 1093.57 & 1084.002 & 1086.4 & 1086.4 & 1004600 \\
\hline $1 / 5 / 2018$ & 1094 & 1104.25 & 1092 & 1102.23 & 1102.23 & 1279100 \\
\hline $1 / 8 / 2018$ & 1102.23 & 1111.27 & 1101.62 & 1106.94 & 1106.94 & 1047600 \\
\hline $1 / 9 / 2018$ & 1109.4 & 1110.57 & 1101.231 & 1106.26 & 1106.26 & 902500 \\
\hline $1 / 10 / 2018$ & 1097.1 & 1104.6 & 1096.11 & 1102.61 & 1102.61 & 1042800 \\
\hline $1 / 11 / 2018$ & 1106.3 & 1106.525 & 1099.59 & 1105.52 & 1105.52 & 978300 \\
\hline $1 / 12 / 2018$ & 1102.41 & 1124.29 & 1101.15 & 1122.26 & 1122.26 & 1720500 \\
\hline $1 / 16 / 2018$ & 1132.51 & 1139.91 & 1117.832 & 1121.76 & 1121.76 & 1575300 \\
\hline $1 / 17 / 2018$ & 1126.22 & 1132.6 & 1117.01 & 1131.98 & 1131.98 & 1198700 \\
\hline $1 / 18 / 2018$ & 1131.41 & 1132.51 & 1117.5 & 1129.79 & 1129.79 & 1198200 \\
\hline $1 / 19 / 2018$ & 1131.83 & 1137.86 & 1128.3 & 1137.51 & 1137.51 & 1778200 \\
\hline $1 / 22 / 2018$ & 1137.49 & & & 1155.81 & & 000 \\
\hline $1 / 23 / 2018$ & 1159.85 & 1171.627 & 1158.75 & 1169.97 & 1169.97 & 1333100 \\
\hline $1 / 24 / 2018$ & 1177.33 & 1179.86 & 1161.05 & 1164.24 & 1164.24 & 1416600 \\
\hline $1 / 25 / 2018$ & & & & & & 500 \\
\hline $1 / 26 / 2018$ & 1175.08 & 1175.84 & 1158.11 & 1175.84 & 75.84 & 2018800 \\
\hline $1 / 29 / 2018$ & 1176.48 & 1186.89 & 1171.98 & 1175.58 & 1175.58 & 1378900 \\
\hline $1 / 30 / 2018$ & & & & & & 300 \\
\hline $1 / 31 / 2018$ & 1170.57 & 1173 & 1159.13 & 1169.94 & 1169.94 & 8700 \\
\hline $2 / 1 / 2018$ & 1162.61 & 1174 & 1157.52 & 1167.7 & 1167.7 & 2100 \\
\hline $2 / 2 / 2018$ & & & & & & 900 \\
\hline $2 / 5 / 2018$ & 1090.6 & 1110 & 1052.03 & 1055.8 & 1055.8 & 3798300 \\
\hline $2 / 6 / 2018$ & 1027.18 & 1081.71 & 1023.137 & 1080.6 & 1080.6 & 3448000 \\
\hline $2 / 7 / 2018$ & & & & & & 2369200 \\
\hline $2 / 8 / 2018$ & 1055.41 & 1058.62 & 1000.66 & 01.52 & 1.52 & 2859100 \\
\hline $2 / 9 / 2018$ & 1017.25 & 1043.97 & 992.56 & 1037.78 & 1037.78 & 3505900 \\
\hline $2 / 12 / 2018$ & 1048 & 1061.5 & 040.928 & 1051.94 & 1051.94 & 2057700 \\
\hline $2 / 13 / 2018$ & 1045 & & 1044.087 & 1052.1 & 1052.1 & 1265100 \\
\hline $2 / 14 / 2018$ & 1048.95 & 1071.72 & 1046.75 & 1069.7 & 1069.7 & 1555800 \\
\hline $2 / 15 / 2018$ & 1079.07 & 1091.479 & 1064.34 & 1089.52 & 1089.52 & 1843400 \\
\hline $2 / 16 / 2018$ & & 1104.67 & 1088.313 & 1094.8 & 1094.8 & 1681600 \\
\hline $2 / 20 / 2018$ & 1090.57 & 1113.95 & 1088.52 & 1102.46 & 1102.46 & 1423100 \\
\hline $2 / 21 / 2018$ & 1106.47 & 1133.97 & 1106.33 & 1111.34 & 1111.34 & 1512900 \\
\hline $2 / 22 / 2018$ & 1116.19 & 1122.82 & 1102.59 & 1106.63 & 1106.63 & 1317200 \\
\hline $2 / 23 / 2018$ & 1112.64 & 1127.28 & 1104.714 & 1126.79 & 1126.79 & 1261000 \\
\hline $2 / 26 / 2018$ & 1127.8 & 1143.96 & 1126.695 & 1143.75 & 1143.75 & 1559100 \\
\hline $2 / 27 / 2018$ & 1141.24 & 1144.04 & 1118 & 1118.29 & 1118.29 & 1774100 \\
\hline $2 / 28 / 2018$ & 1123.03 & 1127.53 & 1103.24 & 1104.73 & 1104.73 & 1882600 \\
\hline
\end{tabular}


British Journal of Computer, Networking and Information Technology

ISSN: 2689-5315

Volume 4, Issue 2, 2021 (pp. 12-21)

www.abjournals.org

\begin{tabular}{|c|c|c|c|c|c|c|}
\hline $3 / 1 / 2018$ & 1107.87 & 1110.12 & 1067.001 & 1069.52 & 1069.52 & 2515900 \\
\hline $3 / 2 / 2018$ & 1053.08 & 1081.999 & 1048.115 & 1078.92 & 1078.92 & 2271600 \\
\hline $3 / 5 / 2018$ & 1075.14 & 1097.1 & 1069 & 1090.93 & 1090.93 & 1202200 \\
\hline $3 / 6 / 2018$ & 1099.22 & 1101.85 & 1089.775 & 1095.06 & 1095.06 & 1532800 \\
\hline $3 / 7 / 2018$ & 1089.19 & 1112.22 & 1085.482 & 1109.64 & 1109.64 & 1292500 \\
\hline $3 / 8 / 2018$ & 1115.32 & 1127.6 & 1112.8 & 1126 & 1126 & 1355100 \\
\hline $3 / 9 / 2018$ & 1136 & 1160.8 & 1132.461 & 1160.04 & 1160.04 & 2128000 \\
\hline $3 / 12 / 2018$ & 163.85 & 1177.05 & 1157.42 & 1164.5 & 1164.5 & 2172300 \\
\hline $3 / 13 / 2018$ & 1170 & 1176.76 & 1133.33 & 1138.17 & 1138.17 & 1907200 \\
\hline $3 / 14 / 2018$ & 1145.21 & 1158.59 & 1141.44 & 1149.49 & 1149.49 & 1291400 \\
\hline $3 / 15 / 2018$ & 1149.96 & 1161.08 & 1134.54 & 1149.58 & 1149.58 & 400 \\
\hline $3 / 16 / 2018$ & 1154.14 & 1155.88 & 1131.96 & 1135.73 & 1135.73 & 2000 \\
\hline $3 / 19 / 2018$ & 1120.01 & 1121.99 & 1089.01 & 1099.82 & 1099.82 & 2805900 \\
\hline $3 / 20 / 2018$ & 1099 & 1105.2 & 1083.46 & 1097.71 & 1097.71 & 900 \\
\hline $3 / 21 / 2018$ & 1092.74 & 1106.3 & 1085.15 & 1090.88 & 1090.88 & 900 \\
\hline $3 / 22 / 2018$ & 1081.88 & 1082.9 & 1045.91 & 1049.08 & 1049.08 & 7000 \\
\hline $3 / 23 / 2018$ & 1047.03 & 1063.36 & 1021.22 & 1021.57 & 1021.57 & 5700 \\
\hline $3 / 26 / 2018$ & 1046 & 1055.63 & 1008.4 & 1053.21 & 1053.21 & 5100 \\
\hline $3 / 27 / 2018$ & 1063 & 1064.839 & 996.92 & 1005.1 & 1005.1 & 5300 \\
\hline $3 / 28 / 2018$ & 998 & 1024.23 & 980.64 & 1004.56 & 1004.56 & 9300 \\
\hline $3 / 29 / 2018$ & 1011.63 & 1043 & 1002.9 & 1031.79 & 1031.79 & 2726800 \\
\hline $4 / 2 / 2018$ & 1022.82 & 1034.8 & 990.37 & 1006.47 & 1006.47 & 2680400 \\
\hline $4 / 3 / 2018$ & 1013.91 & 1020.99 & 994.07 & 1013.41 & 1013.41 & 5100 \\
\hline $4 / 4 / 2018$ & 993.41 & 1028.718 & 993 & 1025.14 & 1025.14 & 2484700 \\
\hline $4 / 5 / 2018$ & 1041.33 & & 1020.131 & & & 1363000 \\
\hline $4 / 6 / 2018$ & 1020 & 1031.42 & 1003.03 & 1007.04 & .04 & 1746400 \\
\hline $4 / 9 / 2018$ & 1016.8 & 1039.6 & 1014.08 & 1015.45 & 1015.45 & 1751600 \\
\hline $4 / 10 / 2018$ & 1026.44 & 1036.28 & & 1031.64 & & 1974500 \\
\hline $4 / 11 / 2018$ & 1027.99 & 1031.364 & 1015.87 & 1019.97 & 1019.97 & 1483900 \\
\hline $4 / 12 / 2018$ & 1025.04 & 1040.69 & 1021.435 & 1032.51 & 1032.51 & 1357000 \\
\hline $4 / 13 / 2018$ & 1040.88 & 1046.42 & 1022.98 & 1029.27 & 1029.27 & 1223000 \\
\hline $4 / 16 / 2018$ & 1037 & 1043.24 & 1026.74 & 1037.98 & 1037.98 & 1211200 \\
\hline $4 / 17 / 2018$ & 1051.37 & 1077.88 & 1048.26 & 1074.16 & 1074.16 & 2320300 \\
\hline $4 / 18 / 2018$ & 1077.43 & 1077.43 & 1066.225 & 1072.08 & 1072.08 & 1344100 \\
\hline $4 / 19 / 2018$ & 1069.4 & 1094.165 & 1068.18 & 1087.7 & 1087.7 & 1747700 \\
\hline $4 / 20 / 2018$ & 1082 & 1092.35 & 1069.57 & 1072.96 & 1072.96 & 1889700 \\
\hline $4 / 23 / 2018$ & 1077.86 & 1082.72 & 1060.7 & 1067.45 & 1067.45 & 2341300 \\
\hline $4 / 24 / 2018$ & 1052 & 1057 & 1010.59 & 1019.98 & 1019.98 & 4760300 \\
\hline $4 / 25 / 2018$ & 1025.52 & 1032.49 & 1015.31 & 1021.18 & 1021.18 & 2391100 \\
\hline $4 / 26 / 2018$ & 1029.51 & 1047.98 & 1018.19 & 1040.04 & 1040.04 & 2079500 \\
\hline $4 / 27 / 2018$ & 1046 & 1049.5 & 1025.59 & 1030.05 & 1030.05 & 1619800 \\
\hline $4 / 30 / 2018$ & 1030.01 & 1037 & 1016.85 & 1017.33 & 1017.33 & 1671300 \\
\hline
\end{tabular}


British Journal of Computer, Networking and Information Technology

ISSN: 2689-5315

Volume 4, Issue 2, 2021 (pp. 12-21)

www.abjournals.org

\begin{tabular}{|c|c|c|c|c|c|c|}
\hline $5 / 1 / 2018$ & 1013.66 & 1038.47 & 1008.21 & 1037.31 & 1037.31 & 1427900 \\
\hline $5 / 2 / 2018$ & 1028.1 & 1040.389 & 1022.87 & 1024.38 & 1024.38 & 1603100 \\
\hline $5 / 3 / 2018$ & 1019 & 1029.675 & 1006.29 & 1023.72 & 1023.72 & 1815100 \\
\hline $5 / 4 / 2018$ & 1016.9 & 1048.51 & 1016.9 & 1048.21 & 1048.21 & 1938700 \\
\hline $5 / 7 / 2018$ & 1049.23 & 1061.68 & 1047.1 & 1054.79 & 1054.79 & 1466100 \\
\hline $5 / 8 / 2018$ & 1058.54 & 1060.55 & 1047.145 & 1053.91 & 1053.91 & 1217700 \\
\hline $5 / 9 / 2018$ & 1058.1 & 1085.44 & 1056.365 & 1082.76 & 1082.76 & 2032800 \\
\hline $5 / 10 / 2018$ & 1086.03 & 1100.44 & 1085.64 & 1097.57 & 1097.57 & 1443000 \\
\hline $5 / 11 / 2018$ & 1093.6 & 1101.33 & 1090.91 & 1098.26 & .26 & 700 \\
\hline $5 / 14 / 2018$ & 1100 & 1110.75 & 1099.11 & 1100.2 & 1100.2 & 1518100 \\
\hline $5 / 15 / 2018$ & 1090 & 1090.05 & 1073.47 & 1079.23 & 1079.23 & 900 \\
\hline $5 / 16 / 2018$ & 1077.31 & 1089.27 & 1076.26 & 1081.77 & 1081.77 & 7300 \\
\hline $5 / 17 / 2018$ & 1079.89 & 1086.87 & 1073.5 & 1078.59 & 1078.59 & 800 \\
\hline $5 / 18 / 2018$ & 1061.86 & 1069.94 & 1060.68 & .36 & .36 & 200 \\
\hline $5 / 21 / 2018$ & 1074.06 & 1088 & 1073.65 & .58 & 9.58 & 200 \\
\hline $5 / 22 / 2018$ & 1083.56 & 1086.59 & 5.69 & 1069.73 & 9.73 & 000 \\
\hline $5 / 23 / 2018$ & 1065.13 & 1080.78 & .71 & 69 & .69 & 000 \\
\hline $5 / 24 / 2018$ & 1079 & 1080.47 & 1066.15 & 1079.24 & .24 & 756800 \\
\hline $5 / 25 / 2018$ & 1079.02 & 1082.56 & 1073.775 & .66 & .66 & 400 \\
\hline $5 / 29 / 2018$ & 1064.89 & 1073.37 & 1055.22 & 1060.32 & 1060.32 & 1856900 \\
\hline $5 / 30 / 2018$ & 1063.03 & 1069.21 & 1056.83 & 1067.8 & 1067.8 & 500 \\
\hline $5 / 31 / 2018$ & 1067.56 & 1097.19 & 1067.56 & 108 & .99 & 300 \\
\hline $6 / 1 / 2018$ & 1099.35 & 1120 & 8.5 & 19.5 & 1119.5 & 2412000 \\
\hline $6 / 4 / 2018$ & 1122.33 & 1141.89 & 1122.005 & 1139.29 & 1139.29 & 1880000 \\
\hline $6 / 5 / 2018$ & 1140.99 & 1145.738 & 1133.19 & 1139.66 & 1139.66 & 3000 \\
\hline $6 / 6 / 2018$ & 1142.17 & 1143 & 1125.743 & 1136.88 & .88 & 1698200 \\
\hline $6 / 7 / 2018$ & 1131.32 & 1135.82 & .52 & .86 & 1123.86 & 1520000 \\
\hline $6 / 8 / 2018$ & 1118.18 & 1126.67 & 1112.15 & 1120.87 & 1120.87 & 1290800 \\
\hline $6 / 11 / 2018$ & 1118.6 & & 1118.6 & 1129.99 & 1129.99 & 1079300 \\
\hline $6 / 12 / 2018$ & 1131.07 & 1139.79 & 1130.735 & 1139.32 & 1139.32 & 912000 \\
\hline $6 / 13 / 2018$ & 1141.12 & 1146.5 & 1133.38 & 1134.79 & 1134.79 & 1506400 \\
\hline $6 / 14 / 2018$ & 1143.85 & 1155.47 & 1140.64 & 1152.12 & 1152.12 & 1343400 \\
\hline $6 / 15 / 2018$ & 1148.86 & 1153.42 & 1143.485 & 1152.26 & 1152.26 & 2122500 \\
\hline $6 / 18 / 2018$ & 1143.65 & 1174.31 & 1143.59 & 1173.46 & 1173.46 & 1413700 \\
\hline $6 / 19 / 2018$ & 1158.5 & 1171.27 & 1154.01 & 1168.06 & 1168.06 & 1621000 \\
\hline $6 / 20 / 2018$ & 1175.31 & 1186.286 & 1169.16 & 1169.84 & 1169.84 & 1648500 \\
\hline $6 / 21 / 2018$ & 1174.85 & 1177.295 & 1152.232 & 1157.66 & 1157.66 & 1238100 \\
\hline $6 / 22 / 2018$ & 1159.14 & 1162.497 & 1147.26 & 1155.48 & 1155.48 & 1311000 \\
\hline $6 / 25 / 2018$ & 1143.6 & 1143.91 & 1112.78 & 1124.81 & 1124.81 & 2157300 \\
\hline $6 / 26 / 2018$ & 1128 & 1133.21 & 1116.659 & 1118.46 & 1118.46 & 1563200 \\
\hline $6 / 27 / 2018$ & 1121.34 & 1131.836 & 1103.62 & 1103.98 & 1103.98 & 1293900 \\
\hline $6 / 28 / 2018$ & 1102.09 & 1122.31 & 1096.01 & 1114.22 & 1114.22 & 1072400 \\
\hline $6 / 29 / 2018$ & 1120 & 1128.227 & 1115 & 1115.65 & 1115.65 & 1315100 \\
\hline
\end{tabular}


British Journal of Computer, Networking and Information Technology

ISSN: $2689-5315$

Volume 4, Issue 2, 2021 (pp. 12-21)

www.abjournals.org

\section{DISCUSSION AND RESULT}

Python is used to implement this system. From Fig 1, we noticed a very significance increase in the stock price in the year 2018 and their decrease afterwards down to asignificante system is capable of predicting stock price.

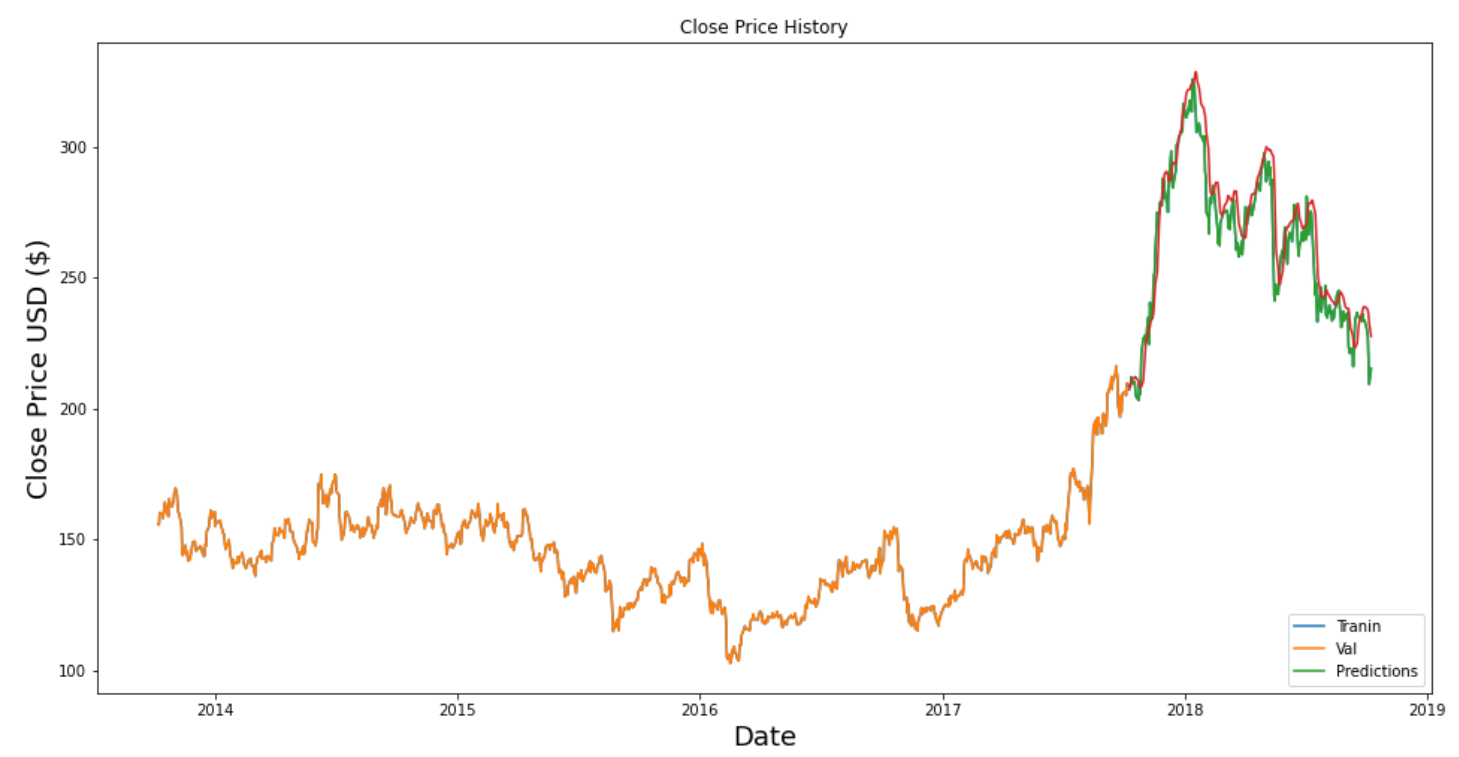

FIG 1: Stock Market Prediction

\begin{tabular}{|r|r|r|}
\multicolumn{1}{l|}{ Date Close } & \multicolumn{1}{c|}{ Predictions } \\
\hline 2017-10-09 & 208.3 & 207.136383 \\
\hline $\mathbf{2 0 1 7 - 1 0 - 1 0}$ & 208.45 & 208.315948 \\
\hline $\mathbf{2 0 1 7 - 1 0 - 1 1}$ & 209.4 & 209.207306 \\
\hline $\mathbf{2 0 1 7 - 1 0 - 1 2}$ & 212.0 & 209.972534 \\
\hline $\mathbf{2 0 1 7 - 1 0 - 1 3}$ & 210.25 & 210.973175 \\
\hline $\mathbf{\cdots}$ & $\ldots$ & \\
\hline $\mathbf{2 0 1 8 - 1 0 - 0 1}$ & 230.9 & 238.434036 \\
\hline $\mathbf{2 0 1 8 - 1 0 - 0 3}$ & 227.6 & 237.555710 \\
\hline $\mathbf{2 0 1 8 - 1 0 - 0 4}$ & 218.2 & 235.966064 \\
\hline $\mathbf{2 0 1 8 - 1 0 - 0 5}$ & 209.2 & 232.669464 \\
\hline $\mathbf{2 0 1 8 - 1 0 - 0 8}$ & 215.15 & 227.5667 \\
\hline
\end{tabular}




\section{CONCLUSION}

Predicting stock prices with some degree of accuracy is critical, especially because it assists business owners or investors in making sound decisions. Although accurate forecasting requires a high level of programming language and machine learning. Our analysis in this paper shows a significant correlation between the yearly prices until the year 2018 where there is a significant increase in stock price. Despite the fact that there are several challenges in our program and that our data collection can be improved, more research can be done, particularly to have refined search data and a better algorithm.

\section{REFERENCES}

Burton, G. M. (1973). A random walk down wall street. Retrieved from https://www.researchgate.net/publication/325247657_Burton_G_Malkiel's_A_random_ walk_down_wall_street

Donald, S. R. (2015). Digital Signal Processing Using the ARM Cortex M4 1st Edition. Retrieved from https://www.amazon.com/Digital-Signal-Processing-UsingCortex/dp/1118859049

Fama, E., \& French, K. (1992). The Cross-Section of Expected Stock Returns. The Journal of Finance, 47(2), $427-465$.

Gardner, W. A. (1992). A unifying view of coherence in signal processing. Retrieved from https://www.sciencedirect.com/science/article/abs/pii/016516849290015O

Ibuomo, R. T. (2020) Digital Signal Processing for Predicting Stock Prices Using IBM Cloud Watson Studio. Retrieved from

http://www.internationaljournalssrg.org/IJCSE/2020/Volume7-Issue1/IJCSEV7I1P102.pdf

Ledisi, G. K., \& Minafa, T. D. (2020). Digital Signal Processing for Predicting Stock Prices in Financial market. Journal of Environmental Science, Computer Science and Engineering \& Technology, 9(2), 196-203.

Michael, B. (2020). Introduction: Predictive Analytics for Stock Price Prediction. Retrieved from https://insightincmiami.org/building-a-stock-market-prediction-model/

Okpor, M. D. (2020). Digital Signal Processing for Predicting Stock Prices. International Journal of Computer Applications, 175 (26), 0975 - 8887.

Sharma, S., \& Kaushik, B. (2018). Quantitative Analysis of Stock Market Prediction for Accurate Investment Decisions in Future, Journal of Artificial Intelligence, 11 (1), 48 54.

ShashankIyer, A., Nisarg, R. K., \& Bahar, S. (2015). Stock Market Prediction using Digital Signal Processing Models. International Journal of Computer Applications, 29(2), 0975 $-8887$.

Shivanker, D. D., Geeta, N., \& Poonam, P. (2013). "Isolated Speech Recognition using MFCC and DTW", International Journal of Advanced Research in Electrical, Electronics and Instrumentation Engineering, 2278-8875. 
British Journal of Computer, Networking and Information Technology

ISSN: 2689-5315

Volume 4, Issue 2, 2021 (pp. 12-21)

www.abjournals.org

Singh, A. (2018). Stock Prices Prediction Using Machine Learning and Deep Learning Techniques (with Python codes). Retrieved from https://www.analyticsvidhya.com/blog/2018/10/predicting-stock-price-machinelearningnd-deep-learning-techniques-python/

Syed, S. N. (2018). Digital Signal Processing for Predicting Stock Prices. Retrieved from https://medium.com/@ sadatnazrul/digital-signal-processing-for-predicting-stock-prices4be247a09514

Wikipedia (2009). Digital Signal Processing. Retrieved from https://en.wikipedia.org/wiki/Digital_signal_processing 